\title{
Screening for Obstructive Sleep Apnea using Epworth Sleepiness Score and Berlin Questionnaire: Which is Better?
}

\author{
Ramakant Dixit, Satyadeep Verma, Kamender Singh Pawar \\ Department of Respiratory Medicine, JLN Medical College, Ajmer, Rajasthan, India
}

\section{Abstract}

Background: Obstructive sleep apnea (OSA) is a highly prevalent, but underdiagnosed and undertreated disorder. There is a need for simple but accurate tool for early detection of patients based on their clinical symptoms and physical findings into high- or low-risk group and give information for urgent polysomnography (PSG) and further treatment to prevent serious consequences. Epworth Sleepiness Score (ESS) and Berlin Questionnaire (BQ) are the most popular and widely acceptable instruments for identification of high-risk patients of OSA. The aim of our study was to compare these two established and well-known sleep questionnaires regarding their ability to find out the probable cases of OSA in Indian scenario. Methods: This cross-sectional study was conducted at a tertiary care center on 72 adult patients with symptoms of sleep-related breathing disorders. All patients were asked to fill both ESS and BQ questionnaires. Subsequently, all patients were subjected to level-1 PSG. For each patient, the Apnea-hypopnoea index was calculated to assess the diagnosis and severity of OSA and to further compare the ESS and BQ questionnaires for their sensitivity (SN) in OSA patients. Results: A total of 14 (19.4\%) patients were diagnosed as OSA by ESS while by BQ, $32(44.4 \%)$ patients were diagnosed as having OSA with SN of $31 \%$ and $71.11 \%$, respectively $(P=0.00004)$. The positive predictive value was $82.3 \%$ and $88.8 \%$ for ESS and BQ, respectively, with a negative predictive value of $43.6 \%$ by ESS and $63.8 \%$ by BQ questionnaires. Conclusion: ESS is a less sensitive diagnostic tool for early detection of high-risk patients of OSA in general population. BQ is a valid, reliable, and more sensitive parameter to screen patients for OSA and may help in improving the quality of life in such patients with proper OSA management.

Keywords: Apnea-Hypopnea Index, body mass index, day-time sleepiness, obstructive sleep apnea, screening tools

\section{INTRODUCTION}

Obstructive sleep apnea (OSA) is a common sleep disorder characterized by repeated episodes of upper airway collapse that results in brief periods of breathing cessation (apnea) or a marked reduction in airflow (hypopnea) during sleep. ${ }^{[1]}$ This condition is associated with loud snoring, oxygen desaturation, and frequent arousal from sleep. These effects are reflected as impaired concentration during day time, increased incidence of accidents, and poor quality of life. ${ }^{[2]}$ The global prevalence of OSA in males is $2 \%-4 \%$, while in females, it is $1 \%-2 \%{ }^{[3]}$ In Indian scenario, the prevalence of OSA is similar at $2.4 \%-4.9 \%$ in males and $1 \%-2 \%$ in females. ${ }^{[4]}$

The gold standard test for diagnosis of OSA is Level 1 polysomnography (PSG). However, the occurrence of OSA is more prevalent than the availability of sleep laboratory. It is a time-consuming and expensive procedure also. Therefore, the evaluation of screening tool becomes necessary to classify

\begin{tabular}{|l|l|}
\hline \multicolumn{2}{|c|}{ Access this article online } \\
\hline Quick Response Code: & Website: \\
& www.ijrconline.org \\
& \\
\end{tabular}

patients based on their clinical symptoms, physical findings, risk factors, and comorbid illness into high- or low-risk group and gives an idea about an urgent need for PSG and further treatment.

A number of screening questionnaires and clinical screening models have been developed to help identify patients with OSA. Among them, Epworth Sleepiness Score (ESS) ${ }^{[5]}$ and Berlin Questionnaire (BQ) $)^{[6]}$ are the most popular and widely used sleep questionnaires. ESS, created by Murray Johns in 1990 , is a validated self-administrated, 8-item questionnaire that measures subjective day-time sleepiness. The BQ was developed in 1996 at the Conference on Sleep in Primary Care

Address for correspondence: Prof. Ramakant Dixit, Department of Respiratory Medicine, JLN Medical College, Ajmer - 305 001, Rajasthan, India. E-mail: dr.ramakantdixit@gmail.com

This is an open access article distributed under the terms of the Creative Commons Attribution-NonCommercial-ShareAlike 3.0 License, which allows others to remix, tweak, and build upon the work non-commercially, as long as the author is credited and the new creations are licensed under the identical terms.

For reprints contact: reprints@medknow.com

How to cite this article: Dixit R, Verma S, Pawar KS. Screening for obstructive sleep apnea using Epworth Sleepiness score and Berlin Questionnaire: Which is better? Indian J Respir Care 2018;7:33-6. 
in Berlin, Germany. It is a validated instrument that is used to identify individuals who are at risk for OSA in primary and some nonprimary care settings.

Although these questionnaires were developed many years ago, their validity to screen out patients for OSA is Effectious. Every questionnaire has its own advantages and disadvantages. The aim of this study was to compare these two established and well-known sleep questionnaires regarding their ability to detect probable cases of OSA in the Indian scenario.

\section{Methods}

This was a cross-sectional study conducted on adult patients presenting with signs, symptoms, and history suggestive of OSA and willing to participate in the study. Institutional ethical committee's approval was obtained. The enrolled patients were assessed by detailed clinical history, vital parameters including blood pressure (BP), clinical assessment for OSA based on day-night symptoms, and anthropometric measurements such as body mass index (BMI).

BMI was calculated using the following formula: $\mathrm{BMI}=\frac{\text { Weight }(\mathrm{kg})}{\operatorname{Height}\left(\mathrm{m}^{2}\right)}$

A BMI of 18.5-24.9 was considered normal, 25-29.9 as overweight, and 30-34.9 as obese. The patients were asked to fill both the ESS and BQ questionnaires.

\section{Epworth Sleepiness score}

The ESS is a self-administered questionnaire with eight questions. It measures the general level of day-time sleepiness in a given person.

In this test, patients are asked to rate, on a 4-point scale (0-3), their usual chances of dozing off or falling asleep in eight different situations or activities that are experienced by most patients in their daily routine work. This basically needs a mental judgment by the study participant. The total ESS score is the sum of 8 scores that can range between 0 and 24; scores $>10$ are usually associated with excessive day-time sleepiness and are considered to be at high risk for OSA. ${ }^{[5]}$

The total ESS score provides an estimate of a general characteristic of each participant and his/her average level of sleepiness in daily activities. It is not a diagnostic tool in itself but very useful to measure sleep-wake health status of an individual. There are other subjective and objective methods also for measuring the overall sleepiness, but the ESS has an edge over others in view of its cost-effectiveness and is a very simple tool to administer with large numbers of participants.

\section{Berlin Questionnaire}

In this questionnaire, there are ten questions separated into three categories. The first category comprises five questions related to snoring, witnessed apneas, and the frequency of such events. The second category comprises three questions that address the day-time sleepiness. The last one and third category comprises two questions regarding the history of high $\mathrm{BP}(>140 / 90 \mathrm{mmHg})$ and BMI of $>30 \mathrm{~kg} / \mathrm{m}^{2}$. The overall BQ results are based on the response from these three categories. Categories 1 and 2 are considered to be positive if there are $>2$ positive responses to each category, while category 3 is considered positive when there is self-reporting of high BP and/or a BMI of $>30 \mathrm{~kg} / \mathrm{m}^{2}$. The study participants are scored as being at "high risk" of having OSA if scores are positive for two or more of these three categories. Participants positive for $<2$ categories are considered as "low risk" for OSA. ${ }^{[6]}$

All the enrolled patients were subjected to the overnight PSG (Level 1) at sleep laboratory of our department using Alice PDx system, manufactured by Philips Respironics. Various parameters monitored during PSG were electroencephalogram, electrocardiogram, electromyogram, electro-oculogram, nasal pressure sensor, pulse oximetry, etc., The Apnea-Hypopnea Index (AHI) was calculated using the following formula:

$\mathrm{AHI}=\frac{\text { Total no. of apneas }+ \text { total no. of hypopneas }}{\text { Total sleep time (in hours) }}$

Based on the AHI, the OSA was classified as following: $\mathrm{OSA}=\mathrm{AHI}>5 / \mathrm{h}$ and non-OSA $=\mathrm{AHI}<5 / \mathrm{h}$.

\section{Statistical analysis}

The data were entered using Microsoft Excel sheet. Data of different groups were compared using Chi-square test and $P<0.05$ was considered statistically significant. The diagnostic accuracy, specificity (SP), sensitivity (SN), negative predictive value (NPV), and positive predictive value (PPV) were also calculated.

\section{RESULTS}

A total of 72 patients constituted the study population. Most of the patients (78\%) were between the age group of 30 and 60 years. The mean age of patients was 48.16 years. The male:female ratio was $1: 1.2$. In this study, 14 patients $(19.44 \%)$ had ESS score $>10$ and 32 patients $(44.44 \%)$ belonged to high-risk group according to BQ. A total of 45 patients $(62.5 \%)$ had AHI $>5$ events/h on level 1 PSG. The total number of patients diagnosed as OSA by ESS was 14 (31\%) (true positive), while using BQ, 32 patients $(71.11 \%)$ were diagnosed as having OSA,

\begin{tabular}{|c|c|c|c|}
\hline \multicolumn{4}{|c|}{$\begin{array}{l}\text { Table 1: Comparison of sleep questionnaires in } \\
\text { obstructive sleep apnea and nonobstructive sleep apnea } \\
\text { patients }\end{array}$} \\
\hline $\begin{array}{l}\text { Sleep } \\
\text { questionnaires }\end{array}$ & $\begin{array}{c}\text { OSA } \\
(n=45), n(\%)\end{array}$ & $\begin{array}{c}\text { No OSA } \\
(n=27), n(\%)\end{array}$ & $P$ \\
\hline \multicolumn{4}{|l|}{ ESS } \\
\hline$>10$ & $14(31)$ & $3(11.11)$ & 0.06 \\
\hline$<10$ & $31(68.88)$ & $24(88.88)$ & \\
\hline \multicolumn{4}{|l|}{ BQ } \\
\hline High & $32(71.11)$ & $4(14.81)$ & 0.00004 \\
\hline Low & $13(28.88)$ & $23(85.18)$ & \\
\hline
\end{tabular}


the difference between the two groups was statistically very significant $(P=0.00004)$. The number of false-negative (SP) patients diagnosed by ESS was $31(68.88 \%)$, which was more compared with that of BQ $(n=13,28.88 \%)$ [Table 1].

Table 2 shows different AHI values in patients having OSA and their comparison with ESS. ESS $>10$ was observed in $23.6 \%$ cases and $<10$ in $76.3 \%$ cases. The correlation between different AHI values and ESS values in the two groups (ESS $<10$ and $>10$ ) was compatible for mild and severe OSA cases than those with moderate OSA. However, this difference was statistically not significant $(P=0.06)$. Table 3 shows an overall significant relationship between high-risk cases as per BQ with AHI value $(P=0.00004)$.

In the present study, SN of ESS was $31.1 \%$ and SP was $88.8 \%$ while SN of BQ was $71.1 \%$ and SP was $85 \%$. The PPV was $82.3 \%$ and $88.8 \%$ for ESS and BQ, respectively, with a NPV of $43.6 \%$ by ESS and $63.8 \%$ by BQ.

\section{Discussion}

In this study, we compared two most commonly used standard screening tools, i.e., ESS and BQ, to diagnose OSA in a rapid and most simple way. These questionnaires were proposed among the study patients and scores were evaluated against the PSG-based AHI values, which is a gold standard for the diagnosis of OSA.

Fourteen patients (31\%) were diagnosed as OSA by ESS and 32 patients $(71.1 \%)$ by BQ in the present study. A study on 55 patients conducted by Madhavan et al.$^{[7]}$ found $35(63.63 \%)$ OSA cases by ESS and $47(85.45 \%)$ OSA cases by BQ, with a SN of $67.2 \%$ by ESS and $89 \%$ by BQ for the diagnosis of OSA. In another study on 100 patients by Khaledi-Paveh et al. ${ }^{[8]}$ in 2015, 65 patients were at "high risk" for OSA with SN of $77.3 \%$ by BQ. In larger group studies on 234 patients by Hassan ${ }^{[9]}$ in 2012, four sleep questionnaires were compared

\begin{tabular}{|c|c|c|c|}
\hline AHI & ESS $>10(n=17)$ & ESS $<10(n=55)$ & $P$ \\
\hline$<5$ & 3 & 24 & \\
\hline $5-15$ & 4 & 10 & 0.06 \\
\hline $15-30$ & 1 & 6 & \\
\hline$>30$ & 9 & 15 & \\
\hline
\end{tabular}

ESS: Epworth Sleepiness Score, AHI: Apnea-Hypopnea index

Table 3: High- and low-risk Berlin Questionnaire results with different Apnea-Hypopnea Index values

\begin{tabular}{lccc}
\hline AHI & High $(\boldsymbol{n}=\mathbf{3 6})$ & Low $(\boldsymbol{n}=\mathbf{3 6})$ & $\boldsymbol{P}$ \\
\hline$<5$ & 4 & 23 & \\
$5-15$ & 4 & 6 & 0.00004 \\
$15-30$ & 5 & 4 & \\
$>30$ & 23 & 3 & \\
\hline
\end{tabular}

AHI: Apnea-Hypopnea Index and it was found that the resultant SN of ESS was $72.55 \%$ and that of BQ was $95.07 \%$. Gus et al., ${ }^{[10]}$ Kiciński et al., ${ }^{[11]}$ and Chiu et al. ${ }^{[12]}$ also observed similar results. In a study on chronic obstructive pulmonary disease (COPD) patients by Faria et al. ${ }^{[13]} 15.4 \%$ patients had a high probability of OSA by Sleep Apnea Clinical Score, $32.5 \%$ patients had a high probability by BQ and $40.7 \%$ patients had excessive diurnal somnolence by ESS. According to this study, BQ and ESS did not accurately predict OSA in COPD patients of the study population. The variation that occurred in ESS and BQ in this study was due to the fact that, all patients were nonobese and some of them were having more severe form of disease which is often associated with malnutrition.

Our study demonstrates that ESS had low SN to predict OSA. Also, the numbers of false negatives with ESS were $>2$ times than BQ (31 vs. $13 ; 68.88 \%$ vs. $28.88 \%$ ). This may be possibly due to the fact that ESS is a subjective test and totally dependent on patient's history. It only measures excessive day-time sleepiness of patients which can occur commonly in obese individuals even without OSA and driven by mechanisms other than night-time sleep deprivation. Moreover, Osman et al. had previously demonstrated that ESS is of no value to differentiate between OSA and simple snorers. ${ }^{[14]}$ It is also to be noted that, although globally accepted, some ESS components may not be applicable to all regions, especially in developing countries where modification may be needed to suit the purpose in view of local conditions.

In comparison to ESS, BQ is an objective test, serving it a more diagnostic, valuable, and sensitive parameter for OSA. It includes BP and BMI, which are important risk factors for OSA. Since obesity is a direct risk factor for the development of OSA and is unrelated to patient's own assessment, BQ becomes a more important and superior tool.

\section{Conclusion}

We conclude with a remark that ESS is a less sensitive and poor predictor tool for diagnosis of OSA as comparison to B.Q. More false-negative results occur with ESS that may lead to increased pool of OSA patients with comorbidities and poor quality of sleep as well as poor quality of life in a significant number of patients. BQ is more sensitive and has predictive parameters for picking up OSA, especially the so-called "occult cases" of OSA and may help in improving the quality of life in such patients with proper OSA management.

\section{Financial support and sponsorship}

Nil.

\section{Conflicts of interest}

There are no conflicts of interest.

\section{REFERENCES}

1. Strohl KP. Obstructive sleep apnea syndrome. In: Goldman L, Bennettt JC, editors. Cecil Textbook of Medicine. Philadelphia: W.B. Saunders Company; 2000. p. 462-6. 


\section{Dixit, et al.: Comparison of ESS and BQ as a screening tool for OSA}

2. Victor LD. Obstructive sleep apnea. Am Fam Physician 1999;60:2279-86.

3. Casale M, Pappacena M, Rinaldi V, Bressi F, Baptista P, Salvinelli F, et al. Obstructive sleep apnea syndrome: From phenotype to genetic basis. Curr Genomics 2009;10:119-26.

4. Prasad CN. Obstructive sleep apnea syndrome- Indian Scenario. Perspect Med Res 2013;1:22-5.

5. Johns MW. A new method for measuring daytime sleepiness: The Epworth Sleepiness Scale. Sleep 1991;14:540-5.

6. Thurtell MJ, Bruce BB, Rye DB, Newman NJ, Biousse V. The Berlin questionnaire screens for obstructive sleep apnea in idiopathic intracranial hypertension. J Neuroophthalmol 2011;31:316-9.

7. Madhavan C, Das S, Suganthi B, Kisku KH. The Berlin questionnaire is a more sensitive tool than the Epworth Sleepiness Scale for screening obstructive sleep apnea. Int J Biomed Res 2016;7:122-4.

8. Khaledi-Paveh B, Khazaie H, Nasouri M, Ghadami MR, Tahmasian M. Evaluation of Berlin questionnaire validity for sleep apnea risk in sleep clinic populations. Basic Clin Neurosci 2016;7:43-8.

9. Hassan I. Comparison of four sleep questionnaires for screening obstructive sleep apnea. Egypt J Chest Dis Tuberc 2012;61:433-41.
10. Gus M, Gonçalves SC, Martinez D, de Abreu Silva EO, Moreira LB, Fuchs SC, et al. Risk for obstructive sleep apnea by Berlin questionnaire, but not daytime sleepiness, is associated with resistant hypertension: A case-control study. Am J Hypertens 2008;21:832-5.

11. Kiciński P, Przybylska-Kuć SM, Tatara K, Dybała A, Zakrzewski M, Mysliński W, et al. Reliability of the Epworth Sleepiness Scale and the Berlin questionnaire for screening obstructive sleep apnea syndrome in the context of the examination of candidates for drivers. Med Pr 2016;67:721-8.

12. Chiu HY, Chen PY, Chuang LP, Chen NH, Tu YK, Hsieh YJ, et al. Diagnostic accuracy of the Berlin questionnaire, STOP-BANG, STOP, and Epworth sleepiness scale in detecting obstructive sleep apnea: A bivariate meta-analysis. Sleep Med Rev 2017;36:57-70.

13. Faria AC, da Costa CH, Rufino R. Sleep apnea clinical score, Berlin questionnaire, or Epworth Sleepiness Scale: Which is the best obstructive sleep apnea predictor in patients with COPD? Int J Gen Med 2015;8:275-81.

14. Osman EZ, Osborne J, Hill PD, Lee BW. The Epworth Sleepiness Scale: Can it be used for sleep apnoea screening among snorers? Clin Otolaryngol Allied Sci 1999;24:239-41. 